EGU2020-18230

EGU General Assembly 2020

(c) Author(s) 2020. This work is distributed under

the Creative Commons Attribution 4.0 License.

\title{
Experimental study on tsunamis generated by landslides
}

Zhenzhu Meng, Christophe Ancey, and Ivan Maeder

Environmental Hydraulics Laboratory, École Polytechnique Fédérale de Lausanne, CH-1015 Lausanne, Switzerland (zhenzhu.meng@epfl.ch)

Tsunami generated by landslides is one of the major threats to populations in coastal areas. A recent example is the 2017 Nuugaatsiaq tsunami. The village in Greenland was partially swept by a tsunami created by a landslide that fell into the Karrat Fjord.

We carried out laboratory experiments to understand how the wave characteristics are related to the landslide features. Emphasis was put on slide-water interactions and efficiency of momentum transfers between the two media. We manufactured granular slides made of differently sized particles using plasticine clay, whose density is close to that of real-world materials. The granular mixture could be shaped, which made it possible to study how the leading edge's shape affected momentum transfer. The mixture was initially placed in a reservoir upstream of a chute, which entered into a water basin. The angle of chute and water depth were kept constant in all our experiments, whereas the material properties and volume were varied systematically. Wave amplitudes and heights were determined from the free-surface variations, which were recorded using a high-speed camera. The velocity field within the water basin was measured using Particle image velocimetry (PIV).

To compare the waves generated by slides exhibiting different properties, empirical equations for prediction of wave characteristics were used. We discuss the differences between experimental results and predictions based on empirical equations. Among other things, we found that the lower the material's permeability, the larger the wave amplitude. 